\title{
Modifying the healthcare system to accommodate our aging population
}

\author{
Adam Kovacs-Litman (Meds 2018), Michael Hewak (Meds 2016) \\ Faculty Reviewer: Dr Chris Brymer, MSc, MD, FRCPC (Division of Geriatric Medicine)
}

In the last 150 years, advances in the field of medicine have extended the average lifespan in the Western world by over 3 decades ${ }^{1}$ and this trend shows no signs of slowing down. If current forecasts are accurate, a third of all babies born in the Western world today will live past their hundredth birthday. ${ }^{2}$ A century ago, this demographic shift had primarily been due to reduced infant mortality and better infection control, and this trend has continued more recently due to better management of chronic conditions like cancer and heart disease. ${ }^{1}$ Nearly half of provincial/territorial government healthcare spending is allocated to seniors (those 65 years of age and older), but our aging population will only cause this proportion to increase, likely reaching unsustainable heights. ${ }^{3}$ This ongoing demographic shift will force us to balance economic sustainability with the effective care of seniors in hospital, postdischarge, and at end-of-life.

A report by the Canadian Institute for Health Information illustrated the current financial burden of seniors on the healthcare system quite handily. While adults aged 15-64 cost our healthcare system an average $\$ 2286$ per capita annually, health spending on seniors was \$10 742 per capita. Furthermore, the financial cost for those seniors aged 80 years and over cost the system a staggering $\$ 18160$ per capita. ${ }^{5}$ This provides a significant challenge for our healthcare system, as it is projected that over the next 25 years, the proportion of the Canadian population 65 and over will jump from $14 \%$ to $25 \%{ }^{4}$

Although longevity is expensive, technically speaking, it is not age itself that increases healthcare expenditure but rather the number of chronic diseases an individual possesses, which tend to rise with age. Indeed, between $50-60 \%$ of seniors suffer from three or more chronic diseases, compared to $15-30 \%$ of those aged less than 65 years. ${ }^{6}$ Most chronic conditions are self-managed by patients and their families at home until patients are typically in their late 70s. Unfortunately, while hospitals are often exceptional at treating patients with acute conditions, their management of patients who require chronic care is often fraught with inefficiency, fragmentation, and poor outcomes. ${ }^{3,7}$

Alternatively, long-term care (LTC) facilities are structured around the rehabilitation and continued management of patients with these chronic and often deteriorating conditions. ${ }^{8}$ They are institutions designed to fill the gaps created by our hospital-centric system, yet they are woefully underutilized by policy-makers. Though the plan is always to transfer chronic patients to LTC or home care, our system is ill-equipped to meet this growing demand. In Ontario alone there are 2000 waiting in hospital beds for LTC. Of the 1000 of these waiting in acute care hospital beds, three quar- ters will be placed in LTC within 10 months; however 15\% will pass away while on the wait list. And this demand-supply mismatch is only getting worse with the median wait time tripling from 2005 to 2012. ${ }^{9}$ What makes this issue even more frustrating is that one day in a hospital costs the province approximately $\$ 1000$, while comparable LTC costs only $\$ 137.10$ and homecare just $\$ 55.11$. Focused investment in LTC would not only improve patient outcomes for the chronically ill, but also save the healthcare system money by freeing up acute care beds, thereby allowing hospitals to better treat those with acute illness.

Seniors with chronic needs occupying acute care beds is a ubiquitous problem in our healthcare system that will only worsen as our population continues to age. The term "Alternate Level of Care" (ALC) describes this phenomenon-patients who have completed the acute phase of their management in hospital, yet continue to occupy an acute care bed. Currently, seniors comprise $40 \%$ of acute hospital stays in Canada, and of the $85 \%$ of such stays that were designated ALC, nearly half were waiting to be placed in an LTC facility. ${ }^{12}$

At Mount Sinai Hospital in Toronto, a program called the Acute Care for Elders (ACE) Strategy has shown great promise as a model for streamlining senior care and reducing the number of ALC patients. The premise of ACE is to implement a higher level of integration across the multiple modalities of healthcare and social services a patient might be receiving across a variety of locations-namely the home, community, and hospital. For example, part of ACE involves screening seniors who present to the emergency room for risk factors that might predict poor outcomes, then adjusting both in-hospital and postdischarge care accordingly. Specialized inpatient geriatric consultation teams manage issues that contribute to functional decline, and aim to avoid hospitalization altogether if possible. Upon discharge, patients are meticulously followed and receive ongoing homecare via partnerships with local organizations, geriatricians, and geriatric psychiatrists. In just one year, the project saw tangible reductions in average length of hospital stay and hospital readmission rates, and patient satisfaction with this program was polled at $97 \% .^{3}$ The cost and cost effectiveness of the resources required to achieve these gains has not been determined yet, however.

Studies have shown that within 30 days of being discharged from the emergency department, $8 \%$ of seniors are either readmitted to hospital or die, with an additional $16 \%$ returning to the ER. ${ }^{13}$ Integrated care systems such as ACE have been shown to keep seniors at home, reducing stress on the healthcare system by decreasing the quantity and length of hospitalizations, while simulta- 
neously improving the level of access and quality of care received. The first four years of PRISMA, a similar integrated care project in Quebec, found a 50\% reduction in unmet medical needs, a 20\% reduction in ER visits, a significantly reduced number of hospitalizations and a 14\% decrease in individuals experiencing functional decline. ${ }^{14}$ Again, the economic sustainability of this program has not been established.

It is clear that medicine works best when it is integrated as part of a multidisciplinary care system that is organized around the needs of the individual, but integration can come in many forms. Several studies suggest that case managers may be an invaluable tool in facilitating the care of the aging population. These are individuals (often nurses) who coordinate care from different providers, ensure that communication is maintained between members of the patient's healthcare team, and educate patients on what care they require and how to go about getting it. Furthermore they would arrange, coordinate, and oversee the home care for hospitalizable conditions such as pneumonia, congestive heart failure, and COPD. A trial of such a project in Australia involved assigning seniors a case manager to oversee their medical care, and resulted in $21 \%$ fewer ER visits and 28\% fewer hospital admissions. ${ }^{15}$

The nuances of palliative care are another area where the hospital machine seems to fall short. The human condition is in part defined by our remarkable ability to hope even in the face of tremendous adversity, even in the face of hopelessness. Broaching the subject of palliative care seems to run contrary to our nature, forcing both healthcare providers and patients to address the uncomfortable subject of death. However, talking about death demystifies it and allows patient preferences to take shape. A randomized control trial looking at lung cancer patients found that those offered palliative care reported improved quality of life at 12 weeks. ${ }^{16}$ As guidelines continue to emerge and healthcare provider education improves, this is an area where we will continue to improve. ${ }^{17,18}$

There was a time when most people died at home surrounded by their families-familiar faces in a familiar environment. Approximately $64 \%$ of people die in hospitals while another $12 \%$ pass away in LTC facilities. ${ }^{19,20}$ This sadly does not accurately reflect patient preferences, as literature shows that the majority of seniors prefer to die in their own home. ${ }^{3}$

As our population ages, palliative care will give the medical profession a tremendous opportunity to put the dignity back in dying, allowing people to pass away comfortably and with meaning. Talking about palliative care is a first step. The next step is to humanize death by listening to patients, helping them realize and articulate their preferences. This will not only improve the quality of a patient's last few months, but also relieve stresses on hospital resources, allowing for more effective care of other patient populations. The availability and flexibility of hospice and palliative home care, however, is a major factor in whether it is even feasible for end of life to occur at home.

The organizational, economic, and political complexity of implementing such significant shifts in the structure of our healthcare system is daunting, but necessary. As more research is done to elucidate the most cost-effective and patient-centered methods of accommodating the rapidly changing demographic landscape, we expect to see increased funding and manpower being allocated to expanding LTC facilities. Further, we expect a greater focus on the use of programs and case managers that integrate each patient's unique mix of medical and social services. We also anticipate a greater emphasis on patient preference at end-of-life, and the development of better palliative care options. What is certain is that Canada's demographic landscape will see unprecedented change in the coming decades as medicine pushes the limits of the human lifespan beyond what was previously imaginable. The question that remains to be answered is how our healthcare system will manage to adapt to meet our aging nation's rapidly evolving medical needs.

\section{REFERENCES}

1. Christensen K, Doblhammer G, Rau R, Vaupel JW. Ageing populations: the challenges ahead. Lancet. 2009;374(9696):1196-208.

2. Office for National Statistics. Historic and projected data from the period and cohort life tables, 2012-based. London: Office for National Statistics; 2013.

3. Bergman H, Hollander M, Jarrett P et al. Health Care in Canada 2011: A focus on seniors and aging. Ottawa: Canadian Institute for Health Information; 2011.

4. Statistics Canada. Population projections for Canada, provinces and territories: 2009 to 2036. Ottawa: Statistics Canada; 2010.

5. Canadian Institute for Health Information. National health expenditure trends, 1975-2010. Ottawa: Canadian Institute for Health Information; 2010.

6. Denton FT, Spencer BG. Chronic health conditions: changing prevalence in an aging population and some implications for the delivery of health care services. Can J Aging. 2010;29(1):11.

7. Simpson J. Chronic condition: why canada's health care system needs to be dragged into the 21 century. Canada: Allen Lane Canada; 2012.

8. Schulz E, Leidl R, König HH. The impact of ageing on hospital care and long-term care - the example of Germany. Health Policy. 2004;67(1):57-74.

9. McCarter J. 2012 annual report, office of the auditor general of Ontario. Toronto: Office of the Auditor General of Ontario; 2012.

10. Ontario Long Term Care Association. This is long-term care 2014. Markham: Ontario Long Term Care Association; 2014.

11. Boyle T. Long-term health care: a look inside the often-baffling system. Toronto: Toronto Star; 2013.

12. Arkinson J, Berthelot JM, Chen XK et al. Health care in Canada 2009. Ottawa: Canadian Institute for Health Information; 2009.

13. McCusker J, Roberge D, Arnaert A et al. Safe discharge of seniors from the emergency department to the community. Montreal: Canadian Patient Safety Institute; 2008.

14. Hébert R, Raîche M, Dubois MF, N’Deye RG, Dubuc N, Tousignant M. Impact of PRISMA, a coordination-type integrated service delivery system for frail older people in Quebec (Canada): a quasi-experimental study. J Gerontol B Psychol Sci Soc Sci. 2009;Jan;65B(1):107-18.

15. Bird SR, Kurowski W, Dickman GK, Kronborg I. Integrated care facilitation for older patients with complex health care needs reduces hospital demand. Aust Health Rev. 2007;31(3):451-61.

16. Temel JS, Greer JA, Muzikansky A, et al. Early palliative care for patients with metastatic non-small-cell lung cancer. New Engl J Med. 2010;363(8):733-42.

17. Care P. Discussing palliative care with patients. Ann Intern Med. 1999;130:744-749. 
18. Last Acts National Program Office. Means to a better end: a report on dying in America today. Washington DC: Last Acts; 2002.

19. Statistics Canada. Ottawa (ON); c2009. Deaths in hospital and elsewhere, Canada, provinces and territories, annual; c2015 [cited 2015 May 1]. Available from http://cansim2.statgan.gc.ca/cgi-win/cnsmcgi. exe?Lang=E\&CNSM-Fi=CII/CII_l-eng.htm.

20. Canadian Institute for Health Information. Quick stats - Canada's health care statistics. Ottawa: Canadian Institute for Health Information; 2014.

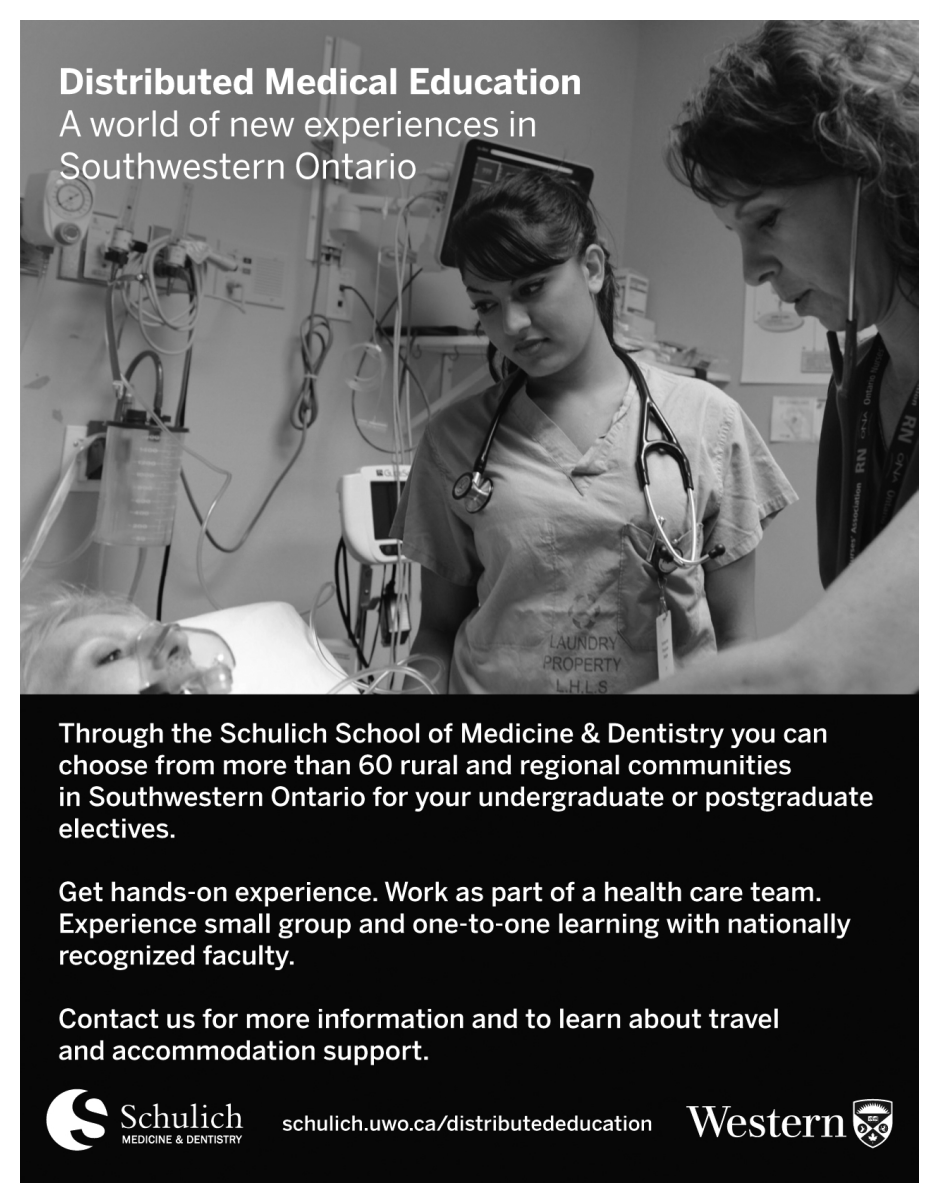

